\title{
Sulcispora supratumida sp. nov. (Phaeosphaeriaceae, Pleosporales) on Anthoxanthum odoratum from Italy
}

\author{
Indunil C. Senanayake ${ }^{1,3,4}$, Rajesh Jeewon ${ }^{5}$, Erio Camporesi ${ }^{6,7,8}, K^{2}$.vin D. Hyde ${ }^{3,4}$, \\ Yu-Jia Zeng², Sheng-Li Tian', Ning Xie'
}

I Shenzhen Key Laboratory of Microbial Genetic Engineering, College of Life Science and Oceanography, Shenzhen University, 3688, Nanhai Avenue, Nanshan, Shenzhen 518055, China 2 Shenzhen Key Laboratory of Laser Engineering, College of Optoelectronic Engineering, Shenzhen University, Shenzhen 518060, China 3 Key Laboratory for Plant Biodiversity and Biogeography of East Asia (KLPB), Kunming Institute of Botany, Chinese Academy of Science, 132 Lanhei Road, Kunming 650201, Yunnan, China 4 Center of Excellence in Fungal Research, Mae Fah Luang University, Chiang Rai 57100, Thailand 5 Department of Health Sciences, Faculty of Science, University of Mauritius, Reduit, 80837, Mauritius 6 A.M.B. Gruppo Micologico Forlivese "Antonio Cicognani", Via Roma 18, Forli, Italy 7 A.M.B. Circolo Micologico "Giovanni Carini", C.P. 314, Brescia, Italy 8 Società per gli Studi Naturalisticidella Romagna, C.P. 144, Bagnacavallo (RA), Italy

Corresponding author: Ning Xie (shainin@msn.cn)

Academic editor: M. Stadler | Received 25 June 2018 | Accepted 27 July 2018 | Published 7 August 2018

Citation: Senanayake IC, Jeewon R, Camporesi E, Hyde KD, Zeng Y-J, Tian S-L, Xie N (2018) Sulcispora supratumida sp. nov. (Phaeosphaeriaceae, Pleosporales) on Anthoxanthum odoratum from Italy. MycoKeys 38: 35-46. https://doi. org/10.3897/mycokeys.38.27729

\begin{abstract}
Sulcispora is typified by $S$. pleurospora. We collected a sulcispora-like taxon on leaves of Anthoxanthum odoratum L. in Italy and obtained single ascospore isolates. Combined ITS, LSU, SSU and tef1 sequence analyses suggested that Sulcispora is placed in the family Phaeosphaeriaceae and a newly collected Sulcispo$r a$ species is introduced here as $S$. supratumida sp. nov. Detailed descriptions and illustrations are provided for Sulcispora supratumida and it is compared with the type species, S. pleurospora.
\end{abstract}

\section{Keywords}

Combined gene analysis, Dothideomycetes, graminicolous fungi, new species, spore septation 


\section{Introduction}

Phaeosphaeriaceae is a highly diverse and large family in the order Pleosporales (Hyde et al. 2013) with more than 42 accepted genera (Hyde et al. 2017; Karunarathna et al. 2017; Wanasinghe et al. 2018). Members of Phaeosphaeriaceae are pathogens or hyper-parasites on living plants and humans and saprobes of decaying plant matter (Tennakoon et al. 2016; Ahmed et al. 2017).

Sulcispora was proposed by Shoemaker and Babcock (1989) as a monotypic genus to accommodate Sulcispora pleurospora (三 Phaeosphaeria pleurospora Niessl). Some morphological characters of Phaeosphaeria pleurospora did not fit within species concepts of Phaeosphaeria and Shoemaker and Babcock (1989), therefore, introduced the genus Sulcispora. The genus name refers to the numerous furrows on the ascospore wall (Shoemaker and Babcock 1989). Sulcispora pleurospora has been reported on monocotyledonous hosts in genera such as Anthoxanthum, Carex, Deschampsia, Sesleria and Tofieldia (Leuchtmann 1984; Shoemaker and Babcock 1989).

In this study, we collected sulcispora-like species associated with leaf spots of Anthoxanthum odoratum in Italy. We compared the morphological characters of our collection with the isotype of Sulcispora pleurospora. Morphologically, our collection differs from the type species of Sulcispora, S. pleurospora. Therefore, we introduce our collection as a new species. Combined ITS, LSU, SSU and tef1 sequence analysis including taxa in Phaeosphaeriaceae indicates that the here-studied fungus grouped with "Phaeosphaeria pleurospora" (CBS 460.84) with high support value.

\section{Methods}

Sample collection, specimen examination and single spore isolation

Specimens were collected from Anthoxanthum odoratum L. from Italy in 2013. They were examined and photographed using a Carl Zeiss Discovery V8 stereo-microscope fitted with Axiocam. Sections of ascomata were taken by hand under a stereo-microscope. Sections and other micro-morphological characters were photographed using a Nikon Eclipse 80i compound microscope fitted with a Canon 450D digital camera. All microscopic measurements were made with Tarosoft image framework (v. 0.9.0.7). Colony characteristics were recorded from cultures grown on Malt Extract Agar (MEA).

Single spore isolation was carried out following the method described by Chomnunti et al. (2014). Germinated ascospores were aseptically transferred into fresh MEA plates and incubated at $20^{\circ} \mathrm{C}$ to obtain pure cultures and later transferred to MEA slants and stored at $4{ }^{\circ} \mathrm{C}$ for further study. The holotype and paratype specimens were deposited at the Mae Fah Luang University (MFLU) fungaria and the herbarium of Kunming Institute of Botany, Chinese Academy of Sciences (HKAS), respectively. Living cultures were deposited at the Mae Fah Luang Culture Collection (MFLUCC). 
MycoBank (http://www.mycobank.org/) and Facesoffungi (Jayasiri et al. 2015) numbers were obtained for the new strain. The new species was established based on recommendations outlined by Jeewon and Hyde (2016).

\section{DNA extraction, PCR amplification and DNA sequencing}

Fresh fungal mycelium grown on MEA for four weeks at $20^{\circ} \mathrm{C}$ was used for DNA extraction (Jeewon et al. 2002). Genomic DNA extraction and PCR reactions were carried out using ITS4/ITS5 for internal transcribed spacer nrDNA (ITS), LR5/LROR for large subunit nrDNA (LSU), NS1/NS4 for large subunit nrDNA (SSU) and $983 \mathrm{~F} / 2218 \mathrm{R}$ for translation elongation factor 1 (tef1) genes according to the same protocol of Maharachchikumbura et al. (2012). The PCR products were observed on 1\% agarose electrophoresis gel stained with ethidium bromide. Purification and sequencing of PCR products were carried out at the Kunming Institute of Botany, Chinese Academy of Science, Kunming, China. Sequence quality was checked and sequences were condensed with DNASTAR Lasergene v.7.1. Sequences derived in this study were deposited in GenBank (Table 1).

\section{Sequence alignment and phylogenetic analysis}

BLASTn searches were made using the newly generated sequences to assist in taxon sampling for phylogenetic analyses. In addition, representatives of the Phaeosphaeriaceae were selected following Tennakoon et al. (2016) and Wanasinghe et al. (2018) (Table 1). Combined multi-locus sequence data of ITS, LSU, SSU and tef1 regions were aligned using default settings of MAFFT v.7 (Katoh et al. 2017) and manually adjusted using BioEdit 7.1.3 (Hall 1999) to allow maximum alignment and minimum gaps. Maximum likelihood analysis was performed by RAxML (Stamatakis and Alachiotis 2010) implemented in raxmlGUIv.1.3 (Silvestro and Michalak 2012). The search strategy was set to rapid bootstrapping and the analysis carried out using the GTRGAMMAI model of nucleotide substitution with 1000 replicates. The model of evolution was estimated by using MrModeltest 2.2 (Nylander 2004).

For the Bayesian inference (BI) analyses of the individual loci and concatenated ITS, LSU, SSU and tef-1 alignment, the above mentioned model test was used to determine the best fitting nucleotide substitution model settings for MrBayes v. 3.0b4. A dirichlet state frequency was predicted for all three data partitions and GTR+I+G as the best model for all single gene and combined datasets. The heating parameter was set to 0.2 and trees were saved every 1000 generations (Ronquist and Huelsenbeck 2003). The Markov Chain Monte Carlo (MCMC) analysis of four chains started in parallel from a random tree topology. The Bayesian analysis lasted 10,000,000 generations (average standard deviation of split frequencies value $=0.0098$ ) and the consensus trees and posterior probabilities were calculated from the 9,998,000 trees sampled 
Table I. Isolates used in this study and their GenBank and culture accession numbers. The strain of Sulcispora supratumida sp. nov. is set in bold font and all ex-type strains are annotated with "T".

\begin{tabular}{|c|c|c|c|c|c|}
\hline Taxon & Culture accession no & ITS & LSU & SSU & tef-1 \\
\hline Allophaeosphaeria muriformia & MFLUCC 13-0349 & KP765680 & KP765681 & KP765682 & - \\
\hline A. subcylindrospora & MFLUCC $13-0380^{\mathrm{T}}$ & KT314184 & KT314183 & KT314185 & - \\
\hline Amarenographium ammophilae & MFLUCC 16-0296 & KU848196 & KU848197 & KU848198 & MG52089 \\
\hline Ampelomyces quisqualis & CBS $129.79^{\mathrm{T}}$ & HQ108038 & JX681064 & EU754029 & - \\
\hline Bhatiellae rosae & MFLUCC 17-0664 & MG828873 & MG828989 & MG829101 & - \\
\hline Chaetosphaeronema hispidulum & CBS 216.75 & KF251148 & KF251652 & EU754045 & - \\
\hline Dactylidina dactylidis & MFLUCC 14-0963 & MG828887 & MG829003 & MG829114 & MG829199 \\
\hline D. shoemakeri & MFLUCC 14-0966 & MG828886 & MG829002 & MG829113 & MG829200 \\
\hline Dematiopleospora mariae & MFLUCC 13-0612 & - & KJ749653 & KJ749652 & KJ749655 \\
\hline Didymella exigua & CBS $183.55^{\mathrm{T}}$ & GU237794 & EU754155 & EU754056 & - \\
\hline Didymocyrtis caloplacae & CBS 1 & JQ238641 & JQ238643 & - & - \\
\hline D. ficuzzae & CBS 128019 & KP170647 & JQ238616 & - & - \\
\hline D. cladoniicola & CBS 128026 & JQ238626 & - & - & - \\
\hline Embarria clematidis & MFLUCC 14-0976 & MG828871 & MG828987 & MG829099 & MG829194 \\
\hline Entodesmium rude & CBS 650.86 & - & GU301812 & - & GU349012 \\
\hline Equiseticola fusispora & MFLUCC 14-0522 & KU987668 & KU987669 & U987670 & MG520895 \\
\hline Galliicola pseudophaeosphaeria & MFLUCC 14-0527 & KT326692 & KT326693 & - & MG829203 \\
\hline Hawksworthiana clematidicola & MFLUCC 14-0910 & MG828901 & MG829011 & MG829120 & MG829202 \\
\hline H. lonicerae & MFLUCC 14-0955 & MG828902 & & MG829121 & MG829203 \\
\hline Italica achilleae & MFLUCC 14-0959 & MG828903 & MG829013 & MG829122 & MG829204 \\
\hline Juncaceicola alpine & CBS 456.84 & & & - & - \\
\hline J. luzulae & FLUCC 16-0780 & KX449529 & KX449530 & X449531 & MG52089 \\
\hline Leptospora rubella & CPC 11006 & DQ195780 & DQ195792 & DQ195803 & - \\
\hline Loratospora aestuarii & JK 5 & - & GU301838 & GU296168 & - \\
\hline L. luzulae & MFLUCC 14-0826 & KT328497 & KT328495 & T328496 & - \\
\hline Melnikia anthoxanthii & MFLUCC $14-1010^{\mathrm{T}}$ & KU848205 & KU848204 & - & - \\
\hline Muriphaeosphaeria gala & MFLUCC 14-0614 & KT438333 & KT438329 & T438331 & MG5209( \\
\hline Neosetophoma italica & MFLUCC14-0826 & KP711356 & KP711361 & KP711366 & - \\
\hline N. samarorum & CBS $138.96^{\mathrm{T}}$ & FJ427061 & KF251664 & GQ387517 & - \\
\hline Neostagonospora caricis & CBS $135092 / S 616^{\mathrm{T}}$ & KF251163 & KF251667 & - & - \\
\hline N. eligiae & CBS $135101^{\mathrm{T}}$ & KF251164 & KF251668 & - & - \\
\hline Nodulosphaeria hirta & MFLUCC 13-0867 & KU708849 & KU708845 & U708841 & KU708853 \\
\hline N. senecionis & MFLUCC 15-1297 & KT290257 & KT290258 & KT290259 & - \\
\hline Ophiobolus cirsii & MFLUCC 13-0218 & KM014664 & KM014662 & KM014663 & - \\
\hline O. disseminans & AS2L14-6 & - & - & KP117305 & - \\
\hline Ophiosphaerella agrostidis & MFLUCC 11-0152 & KM434271 & KM434281 & KM434290 & KM434299 \\
\hline Paraleptosphaeria dryadis & CBS 643.86 & J F740213 & GU301828 & KC584632 & GU349009 \\
\hline Paraphoma chrysanthemicola & CBS 522.66 & FJ426985 & KF251670 & GQ387521 & - \\
\hline P. radicina & CBS $111.79^{\mathrm{T}}$ & KF251172 & KF251676 & EU754092 & - \\
\hline Parastagonospora nodorum & CBS $110109^{\mathrm{T}}$ & KF251177 & KF251681 & EU754076 & - \\
\hline P. poagena & CBS $136776^{\mathrm{T}}$ & KJ869116 & KJ869174 & - & - \\
\hline Phaeosphaeria chiangraina & MFLUCC 13-0231 & KM434270 & KM434280 & KM434289 & KM4342s \\
\hline P. oryzae & CBS $110110^{\mathrm{T}}$ & KF251186 & KF251689 & GQ387530 & - \\
\hline P. papayae & S528 & KF251187 & KF251690 & - & - \\
\hline
\end{tabular}




\begin{tabular}{|c|c|c|c|c|c|}
\hline Taxon & Culture accession no & ITS & LSU & SSU & tef-1 \\
\hline Phaeosphaeria pleurospora & CBS 460.84 & AF439498 & - & - & - \\
\hline $\begin{array}{l}\text { Phaeosphaeriopsis } \\
\text { glaucopunnctata }\end{array}$ & MFLUCC $13-0265^{\mathrm{T}}$ & KJ522473 & KJ522477 & KJ522481 & MG520918 \\
\hline P. triseptata & MFLUCC 13-0271 ${ }^{\mathrm{T}}$ & KJ522475 & KJ522479 & KJ522484 & MG520919 \\
\hline Poaceicola arundinis & MFLUCC 15-0702 & KU058716 & KU058726 & - & MG520921 \\
\hline P. italica & MFLUCC 13-0267 & KX926421 & KX910094 & KX950409 & MG520924 \\
\hline Populocrescntia forlicesesensis & MFLU $15-0651^{\mathrm{T}}$ & KT306948 & KT306952 & KT306955 & MG520925 \\
\hline Premilcurensis senecionis & MFLUCC 13-0575 & KT728365 & KT728366 & - & - \\
\hline Sclerostagonospora sp. & CBS 123538 & FJ372393 & FJ372410 & - & - \\
\hline Scolicosporium minkeviciusii & MFLUCC 12-0089 & - & KF366382 & KF366383 & - \\
\hline Septoriella leuchtmannii & CBS $459.84^{\mathrm{T}}$ & KF251188 & KF251691 & - & - \\
\hline Setomelanomma holmii & CBS 110217 & - & GU301871 & GQ387572 & GU349028 \\
\hline Setophoma sacchari & CBS $333.39^{\mathrm{T}}$ & KF251245 & KF251748 & GQ387525 & - \\
\hline S. terrestris & CBS $335.29^{\mathrm{T}}$ & KF251246 & KF251749 & GQ387526 & - \\
\hline Sulcispora supratumida & MFLUCC 14-0995 & KP271443 & KP271444 & KP271445 & MH665366 \\
\hline Tintelnotia destructans & CBS $127737^{\mathrm{T}}$ & NR_147684 & NG_058274 & KY090698 & - \\
\hline T. destructans & CBS 137534 & - & KY090663 & KY090697 & - \\
\hline Vagicola chlamydospora & MFLUCC 15-0177 & KU163658 & KU163654 & - & - \\
\hline V. vagans & CBS 604.86 & KF251193 & KF251696 & - & - \\
\hline Vrystaatia aloeicola & CBS 135107 & KF251278 & KF251781 & - & - \\
\hline Wojnowicia dactylidis & MFLUCC 13-0735 & KP744470 & KP684149 & KP684150 & - \\
\hline W. lonicerae & MFLUCC 13-0737 & KP744471 & KP684151 & KP684152 & - \\
\hline Wojnowiciella eucalypti & CPC $25024^{\mathrm{T}}$ & KR476741 & KR476774 & - & LT990617 \\
\hline Xenoseptoria neosaccardoi & CBS $128665^{\mathrm{T}}$ & KF251281 & KF251784 & - & - \\
\hline X. neosaccardoi & CBS 120.43 & KF251280 & KF251783 & - & - \\
\hline Yunnanensis phragmitis & MFLUCC $17-0315^{\mathrm{T}}$ & MF684862 & MF684863 & MF684867 & MF683624 \\
\hline Y.phragmitis & MFLUCC $17-1365^{\mathrm{T}}$ & MF684869 & MF684865 & MF684864 & MF683625 \\
\hline
\end{tabular}

CBS: Westerdijk Fungal Biodiversity Institute, Utrecht, The Netherlands; CPC: Culture collection of Pedro Crous, housed at CBS-KNAW; MFLUCC: Mae Fah Luang University Culture Collection, Chiang Rai, Thailand.

after discarding the first $20 \%$ of generations as burn-in. Trees obtained in this study were deposited in TreeBASE under accession number S22938. The phylogram was visualised in FigTree v. 1.2.2 (Rambaut and Drummond 2008).

\section{Results}

\section{Phylogenetic inferences}

The combined ITS, LSU, SSU and tef-1 sequence data set comprised 69 strains of Phaeosphaeriaceae with Didymella exigua as the outgroup taxon. All individual trees generated under different criteria and from single gene datasets were essentially similar in topology and not significantly different from the tree generated from the concat- 
enated dataset. Maximum likelihood analysis with 1000 bootstrap replicates yielded a tree with the likelihood value of $\ln :-13019.593920$ and the following model parameters: alpha: $0.144187 ; \Pi(\mathrm{A}): 0.245356, \Pi(\mathrm{C}): 0.229408, \Pi(\mathrm{G}): 0.267562$ and $\Pi(\mathrm{T})$ : 0.257674 . The best scoring RAxML tree is shown in Figure 1. Maximum likelihood bootstrap values $\geq 50 \%$ and Bayesian inference $(\mathrm{BI}) \geq 0.9$ are given at each node.

The phylogenetic trees obtained from maximum likelihood were topologically congruent to previous studies on Phaeosphaeriaceae (Phookamsak et al. 2014; Thambugala et al. 2014; Tennakoon et al. 2016; Karunarathna et al. 2017; Wanasinghe et al. 2018). This phylogenetic analysis showed the placement of 45 genera within Phaeosphaeriaceae. The here-studied strain clustered with CBS 460.84 (one of Leuchtmann's Swiss strains of S. pleurospora from Carex firma) with 100\% bootstrap support value. The ITS sequence of the CBS 460.84 is almost identical to our strain (MFLUCC 14-0995). However no LSU, SSU and tef-1 sequences were obtained from CBS 460.84 in GenBank. The herbarium specimen of CBS 460.84 is in Westerdijk Fungal Biodiversity Institute (CBS) under accession number CBS H-15991 (SWITZERLAND, Kt. Graubünden, Zügenschlucht near Davos, Carex firma, A. Leuchtmann). However, CBS has presently stopped sending specimens on loan, hence we could not compare morphological characters of the here studied strain with CBS 460.84. Additionally Sulcispora sisterly clustered with the type species of Loratospora, L. aestuarii with low support and the second species of Loratospora, L. luzulae. was distantly clustered.

\section{Taxonomy}

\section{Sulcispora supratumida Senan., Camporesi \& K.D. Hyde, sp. nov.}

MycoBank No: MB826887

Facesoffungi No: FoF 04782

Figure 2

Etymology. The species epithet is based on the two Latin words "supra" meaning upper and "tumidus" meaning swollen, referring to the position of swollen cells of ascospores.

Type. ITALY. Province of Forli-Cesena, Premilcuore, Passodella Valbura, on dead leaves of Anthoxanthum odoratum L. (Poaceae), 25 May 2013, Erio Camporesi, IT 1306 (MFLU 15-0038, holotype; HKAS 83865, paratype): living cultures, MFLUCC 14-0995.

Description. Saprobic on leaves of Anthoxanthum odoratum L., visible as black spots, occurring on the upper surface of entire leaf. Sexual morph. Ascomata 110-150 × 90-140 $\mu \mathrm{m}(\overline{\mathrm{x}}=140-125 \mu \mathrm{m}, \mathrm{n}=10)$, scattered, solitary, immersed, uniloculate, globose, black. Ostiole 35-40 $\mu \mathrm{m}(\overline{\mathrm{x}}=39 \mu \mathrm{m}, \mathrm{n}=10)$ wide, papillate, central, periphysate. Periphyses 15-20 $\mu \mathrm{m}$ long, hyaline. Peridium comprising 2-4 layers of brown to dark brown, thick-walled, cells of textura angularis to textura globularis. Hamathecium comprising 


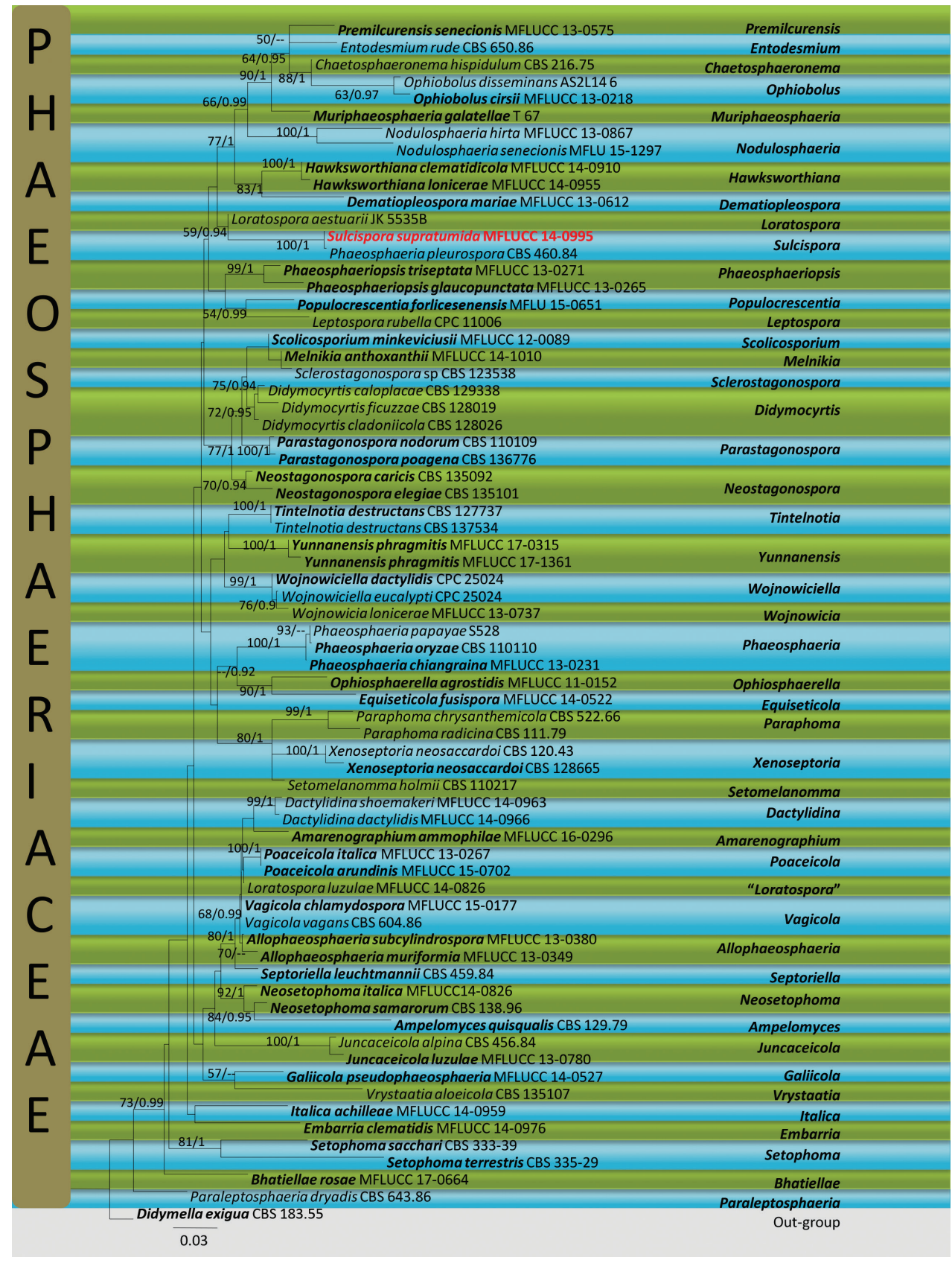

Figure I. Maximum likelihood majority rule consensus tree based on a combined dataset of ITS, LSU, SSU and tef- 1 sequences. Bootstrap support values $\geq 50 \%$ and Bayesian inference $(\mathrm{BI}) \geq 0.9$ are given at the nodes. The tree is rooted to Didymella exigua (CBS 183.55). The culture accession numbers are given after the species names. All ex-type strains are in bold. The newly introduced species from this study is in bold red. 


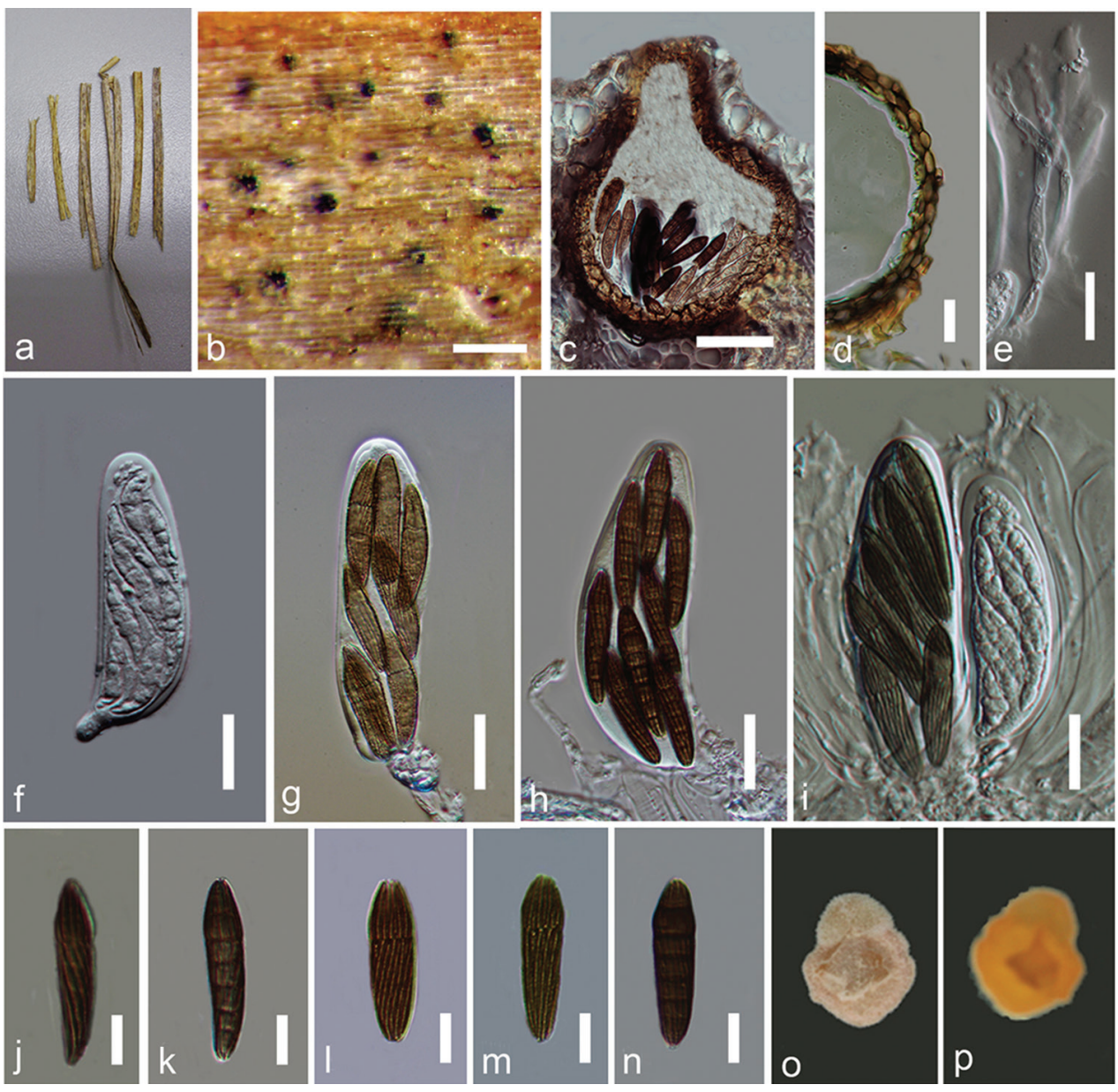

Figure 2. Sulcispora supratumida (MFLU 15-0038). a Leaves of Anthoxanthum odoratum b Appearance of ascomata on host surface $\mathbf{c}$ Cross section of ascoma $\mathbf{d}$ Peridium $\mathbf{e}$ Pseudoparaphyses $\mathbf{f}-\mathbf{i}$ Asci $\mathbf{j}-\mathbf{n}$ Ascospores o Upper surface of the culture $\mathbf{p}$ Lower surface of the culture. Scale bars: $200 \mu \mathrm{m}(\mathbf{b}), 50 \mu \mathrm{m}(\mathbf{c}), 20 \mu \mathrm{m}(\mathbf{d}-\mathbf{i})$, $10 \mu \mathrm{m}(\mathbf{j}-\mathbf{n})$.

2-4 $\mu \mathrm{m}$ wide, cellular, hyaline, branched, septate, pseudoparaphyses, constricted at the septa, anastomosing mostly above the asci and embedded in a mucilaginous matrix. Asci 85-125 $\times 20-35 \mu \mathrm{m}(\overline{\mathrm{x}}=100 \times 30 \mu \mathrm{m}, \mathrm{n}=20), 8$-spored, few, bitunicate, fissitunicate, subglobose to clavate, short pedicellate, apically rounded, with an ocular chamber, arising from the base of the ascoma and attached to parenchymatous cell matrix at base. Ascospores 30-35 $\times 6-9 \mu \mathrm{m}(\overline{\mathrm{x}}=35 \times 7 \mu \mathrm{m}, \mathrm{n}=25)$, bi-seriate to tri-seriate, narrowly fusiform, narrowing towards the end cells, reddish to dark brown, 6-septate, second septum supra-median, slightly constricted, not constricted at other septa, second segment swollen, straight, with 12-16 longitudinal furrows on surface, lacking a mucilaginous sheath. Asexual morph. Undetermined. 
Table 2. Ascospore morphology comparison of Sulcispora species

\begin{tabular}{|c|c|c|c|c|c|}
\hline $\begin{array}{l}\text { Species } \\
\text { name }\end{array}$ & Herbarium type data & Host & $\begin{array}{l}\text { No of } \\
\text { septa }\end{array}$ & \begin{tabular}{|c|}
$\begin{array}{c}\text { Swollen } \\
\text { cell }\end{array}$ \\
\end{tabular} & Reference \\
\hline \multirow{3}{*}{$\begin{array}{l}\text { Sulcispora } \\
\text { pleurospora }\end{array}$} & FH 196419 (isotype) & $\begin{array}{l}\text { Deschampsia cespitosa } \\
\text { (Poaceae) }\end{array}$ & $5-6$ & $3^{\text {rd }}$ & $\begin{array}{l}\text { Shoemaker and } \\
\text { Babcock } 1989\end{array}$ \\
\hline & $\begin{array}{l}\text { F6952, F6949, F6951 } \\
\text { (isotype) }\end{array}$ & $\begin{array}{l}\text { Deschampsia cespitosa } \\
\text { (Poaceae) }\end{array}$ & 6 & $3^{\text {rd }}$ & In this study \\
\hline & $\begin{array}{l}\text { M (1 collection }), \\
\text { ZT (8 collections })\end{array}$ & $\begin{array}{c}6 \text { monocotyledonous hosts, } \\
1 \text { dicotyledonous host }\end{array}$ & $6-8$ & $3^{\text {rd }}$ or $4^{\text {th }}$ & Leuchtmann 1984 \\
\hline \multirow{2}{*}{$\begin{array}{l}\text { Sulcispora } \\
\text { supratumida }\end{array}$} & ZT (6 collections) & $\begin{array}{l}\text { Seleria caerulea (Poaceae) } \\
\text { Carex firma (Cyperaceae) }\end{array}$ & 6 & $2^{\text {nd }}$ & Leuchtmann 1984 \\
\hline & $\begin{array}{l}\text { MFLU 15-0038 } \\
\text { (holotype) }\end{array}$ & $\begin{array}{c}\text { Anthoxanthum odoratum } \\
\text { (Poaceae) }\end{array}$ & 6 & $2^{\text {nd }}$ & In this study \\
\hline
\end{tabular}

Culture characteristics. $2 \mathrm{~cm}$ diameter after 4 weeks incubated in dark at $25^{\circ} \mathrm{C}$ on MEA, pinkish-white, circular, slightly woolly, margin lobate, effuse, lacking aerial mycelium, tightly attached to the media.

\section{Discussion}

Shoemaker and Babcock (1989) observed type specimens of Phaeosphaeria pleurospora and found that the ascospores of $P$. pleurospora with striated ornamented walls are different to those of other genera in Phaeosphaeriaceae. Hence, they introduced the genus Sulcispora to accommodate P. pleurospora and placed it in Phaeosphaeriaceae. Sulcispora pleurospora has some similarities with Phaeosphaeria exarata Shoemaker \& C.E. Babc., in having very large cells in the peridium, ascospores with a continuous sheath and ornamented wall of ascospores with coarse, longitudinal ridges (Shoemaker and Babcock 1989).

In this study, a combined gene sequence analysis of taxa amongst the Phaeosphaeriaceae provides substantial evidence to support Sulcispora as a distinct genus in Phaeosphaeriaceae. Sulcispora differs from other genera in having immersed ascomata with a relatively thin wall, cellular pseudoparaphyses, short pedicellate asci and brown ascospores (Phookamsak et al. 2014).

Leuchtmann (1984) reported variation of ascospore septation amongst several collections of Phaeosphaeria pleurospora from different host plants. Phaeosphaeria pleurospora, collected from Sesleria caerulea (L.) Ard. and Carex firma Mygind ex Host, usually formed 6-septate ascospores and the second segment was swollen. Our collection is morphologically identical to Leuchtmann's collection. However, the isotype and some of Leuchtmann's collections from other host plants had 5-8-septate ascospores and the third or fourth segment was swollen (Table 2). Therefore Leuchtmann (1984) characterised Phaeosphaeria pleurospora as a species with 5-8 septate ascospores. However, Leuchtmann's collection of Sulcispora pleurospora is likely to comprise more than a single species and possibly constitutes a species complex. 
Based on the morphology, we identified our collection as different from the isotype of Sulcispora pleurospora. Hence, we introduced a new species as Sulcispora supratumida sp. nov. However, the ITS sequence of our strain clustered with that of CBS 460.84 (one of Leuchtmann's Swiss strain of S. pleurospora from Carex firma) with 100\% bootstrap support value. There are only two base pair differences between the ITS regions of both strains. Since there are no sequence data of other DNA regions of Sulcispora pleurospora deposited in GenBank, we could not confirm whether or not CBS 460.84 is Sulcispora supratumida. However, it would eventually be practical to obtain the living strain of CBS 460.84 and generate further sequence data.

\section{Keys for species in Sulcispora}

1 Ascomata erumpent, long papillate, 5-8-septated, ascospores with $3^{\text {rd }}$ swollen cell ...................................................................................... pleurospora

- $\quad$ Ascomata immersed, short papillate, 6-septated, ascospores with $2^{\text {nd }}$ swollen cell

S. supratumida

\section{Acknowledgements}

Indunil C. Senanayake thanks to Shaun Pennycook (Landcare Research, Christchurch, New Zealand) for helping to correct this manuscript.

\section{References}

Ahmed SA, Hofmüller W, Seibold M, de Hoog GS, Harak H, Tammer I, van Diepeningen AD, Behrens-Baumann W (2017) Tintelnotia, a new genus in Phaeosphaeriaceae harbouring agents of cornea and nail infections in humans. Mycoses 60(4): 244-253. http://dx.doi. org/10.1111/myc. 12588

Chomnunti P, Hongsanan S, Aguirre-Hudson B, Tian Q, Peršoh D, Dhami MK, Alias AS, Xu J, Liu X, Stadler M, Hyde KD (2014) The sooty moulds. Fungal Diversity 66: 1-36. https://doi.org/10.1007/s13225-014-0278-5

Hall TA (1999) BioEdit: a user-friendly biological sequence alignment editor and analysis program for Windows 95/98/NT. Nucleic Acids Symposium Series 41: 95-98. http://brownlab.mbio.ncsu.edu/JWB/papers/1999Hall1.pdf

Hyde KD, Jones EBG, Liu JK, Ariyawansa HA, Boehm E, Boonmee S, Braun U, Chomnunti P, Crous PW, Dai DQ, Diederich P, Dissanayake A, Doilom M, Doveri F, Hongsanan S, Jayawardena R, Lawrey JD, Li YM, Liu YX, Lücking R, Monkai J, Muggia L, Nelsen MP, Pang KL, Phookamsak R, Senanayake IC, Shearer CA, Suetrong S, Tanaka K, Thambugala KM, Wijayawardene NN, Wikee S, Wu HX, Zhang Y, Aguirre-Hudson B, Alias SA, Aptroot A, Bahkali A, Bezerra JL, Bhat DJ, Camporesi E, Chukeatirote E, Gueidan C, 
Hawksworth DL, Hirayama K, Hoog SD, Kang JC, Knudsen K, Li WJ, Li XH, Liu ZY, Mapook A, Mckenzie EHC, Miller AN, Mortimer PE, Phillips AJL, Raja HA, Scheuer C, Schumm F, Taylor JE, Tian Q, Tibpromma S, Wanasinghe DN, Wang Y, Xu JC, Yacharoen S, Yan JY, Zhang M (2013) Families of Dothideomycetes. Fungal Diversity 63: 1-313. http://dx.doi.org/10.1007/s13225-013-0263-4

Hyde KD, Norphanphoun C, Abreu VP, Bazzicalupo1 A, Chethana KWT, Clericuzio M, Dayarathne MC, Dissanayake AJ, Ekanayaka1 AH, He MQ, Hongsanan S, Huang SK, Jayasiri SC, Jayawardena RS, Karunarathna A, Konta S, Kusan I, Lee H, Li J, Lin CG, Liu NG, Lu YZ, Luo ZL, Manawasinghe IS, Mapook A, Perera RH, Phookamsak R, Phukhamsakda C, Siedlecki I, Soares AM, Tennakoon DS, Tian Q, Tibpromma S, Wanasinghe DN, Xiao YP, Yang J, Zeng XY, Abdel-Aziz FA, Li WJ, Senanayake IC, Shang QJ, Daranagama DA, de Silva NI, Thambugala KM, Abdel-Wahab MA, Bahkali AH, Berbee ML, Boonmee S, Bhat DJ, Bulgakov TS, Buyck B, Camporesi E, Castaneda-Ruiz RF, Chomnunti P, Doilom M, Dovana F, Gibertoni TB, Jadan M, Jeewon R, Jones EBG, Kang JC, Karunarathna SC, Lim YW, Liu JK, Liu ZY, Plautz Jr. HL, Lumyong S, Maharachchikumbura SSN, Matocec N, McKenzie EHC, Mesic A, Miller D, Pawłowska J, Pereira OL, Promputtha I, Romero AL, Ryvarden L, Su HY, Suetrong S, Tkalcec Z, Vizzini A, Wen TC, Wisitrassameewong K, Wrzosek M, Xu JC, Zhao Q, Zhao RL, Mortimer PE (2017) Fungal diversity notes 603-708: taxonomic and phylogenetic notes on genera and species. Fungal Diversity 87: 1-235. https://doi.org/10.1007/s13225-017-0391-3

Jayasiri SC, Hyde KD, Ariyawansa HA, Bhat J, Buyck B, Cai L, Dai YC, Abd-Elsalam KA, Ertz D, Hidayat I, Jeewon R, Jones EBG, Bahkali AH, Karunarathna SC, Liu JK, Luangsa-ard JJ, Lumbsch HT, Maharachchikumbura SSN, McKenzie EHC, Moncalvo JM, GhobadNejhad M, Nilsson H, Pang KA, Pereira OL, Phillips AJL, Raspé O, Rollins AW, Romero AI, Etayo J, Selçuk F, Stephenson SL, Suetrong S, Taylor JE, Tsui CKM, Vizzini A, AbdelWahab MA, Wen TC, Boonmee S, Dai DQ, Daranagama DA, Dissanayake AJ, Ekanayaka AH, Fryar SC, Hongsanan S, Jayawardena RS, Li WJ, Perera RH, Phookamsak R, de Silva NI, Thambugala KM, Tian Q, Wijayawardene NN, Zhao RL, Zhao Q, Kang JC, Promputtha I (2015) The Faces of Fungi database: fungal names linked with morphology, phylogeny and human impacts. Fungal Diversity 74: 3-18. https://doi.org/10.1007/ s13225-015-0351-8

Jeewon R, Hyde KD (2016) Establishing species boundaries and new taxa among fungi: recommendations to resolve taxonomic ambiguities. Mycosphere 7: 1669-1677. https://doi. org/10.5943/mycosphere/7/11/4

Jeewon R, Liew ECY, Hyde KD (2002) Phylogenetic relationships of Pestalotiopsis and allied genera inferred from ribosomal DNA sequences and morphological characters. Molecular Phylogenetics and Evolution 25: 378-392. https://doi.org/10.1016/S10557903(02)00422-0

Karunarathna A, Papizadeh M, Senanayake IC, Jeewon R, Phookamsak R, Goonasekara ID, Wanasinghe DN, Wijayawardene NN, Amoozegar MA, Shahzadeh Fazeli SA, Camporesi E, Hyde KD, Weerahewa HLD, Lumyong S, McKenzie EHC (2017) Novel fungal species of Phaeosphaeriaceae with an asexual/sexual morph connection. Mycosphere $8(10)$ : 1818-1834. https://doi.org/10.5943/mycosphere/8/10/8 
Katoh K, Rozewicki J, Yamada KD (2017) MAFFT online service: multiple sequence alignment, interactive sequence choice and visualization. Briefings in Bioinformatics 108: 1-7. https://doi.org/10.1093/bib/bbx108

Leuchtmann A (1984) Uber Phaeosphaeria Miyake und andere bitunicate Ascomyceten mit mehrfach querseptierten Ascosporen. Sydowia 37: 75-194. https://doi.org/10.3929/ethza-000320965

Maharachchikumbura SSN, Guo LD, Cai L, Chukeatirote E, Wu WP, Sun X, Crous PW, Bhat DJ, McKenzie EHC, Bahkali AH, Hyde KD (2012) A multi-locus backbone tree for Pestalotiopsis, with a polyphasic characterization of 14 new species. Fungal Diversity 56: 95-129. https://doi.org/10.1007/s13225-012-0198-1

Nylander JAA (2004) MrModeltest 2.0. Program distributed by the author. Evolutionary Biology Centre, Uppsala University.

Rambaut A, Drummond A (2008) FigureTree: Tree Figures drawing tool, version 1.2. 2. Institute of Evolutionary Biology, University of Edinburgh.

Ronquist F, Huelsenbeck JP (2003) MrBayes 3: Bayesian phylogenetic inference under mixed models. Bioinformatics 19: 1572-1574. https://doi.org/10.1093/bioinformatics/btg180

Shoemaker A, Babcock E (1989) Phaeosphaeria. Canadian Journal of Botany 67: 1500-1599. https://doi.org/10.1139/b89-199

Silvestro D, Michalak I (2012) raxmlGUI: a graphical front-end for RAxML. Organismic Diversity and Evolution 12: 335-337. https://doi.org/10.1007/s13127-011-0056-0

Stamatakis A, Alachiotis N (2010) Time and memory efficient likelihood-based tree searches on phylogenomic alignments with missing data. Bioinformatics 26: 1132-1139. https://doi. org/10.1093/bioinformatics/btq205

Tennakoon DS, Hyde KD, Phookamsak R, Wanasinghe DN, Camporesi E, Promputtha I (2016) Taxonomy and phylogeny of Juncaceicola gen. nov. (Phaeosphaeriaceae, Pleosporinae, Pleosporales). Cryptogamie, Mycologie 37(2): 135-156. https://doi.org/10.7872/ crym/v37.iss2.2016.135

Thambugala KM, Camporesi E, Ariyawansa HA, Phookamsak R, Liu Z, Hyde KD (2014) Phylogeny and morphology of Phaeosphaeriopsis triseptata sp. nov., and Phaeosphaeriopsis glaucopunctata. Phytotaxa 176(1): 238-250. http://dx.doi.org/10.11646/phytotaxa.176.1.23

Wanasinghe DN, Phukhamsakda C, Hyde KD, Jeewon R, Lee HB, Jones EBG, Tibpromma S, Tennakoon DS, Dissanayake AJ, Jayasiri SC, Gafforov Y, Camporesi E, Bulgakov TS, Ekanayake AH, Perera RH, Samarakoon MC, Goonasekara ID, Mapook A, Li WJ, Senanayake IC, Li JF, Norphanphoun C, Doilom M, Bahkali AH, Xu JC, Mortimer PE, Tibell L, Savic ST, Karunarathna SC (2018) Fungal diversity notes 709-839: taxonomic and phylogenetic contributions to fungal taxa with an emphasis on fungi on Rosaceae. Fungal Diversity 89: 1-236. https://doi.org/10.1007/s13225-018-0395-7 